\title{
Estimating turbulent boundary layer characteristics by high-speed infrared thermography
}

\author{
by E. Koroteeva, I. Znamenskaya, A. Novinskaya
}

\author{
Lomonosov Moscow State University, Leninskie Gory, 1, 119991 Moscow, Russia, koroteeva@physics.msu.ru
}

\begin{abstract}
We propose a new methodology for the quantitative analysis of fluid boundary layers based on high-speed thermographic measurements. The methodology is tested on a boundary-layer flow formed by a single submerged round jet impinging on an infrared-transparent flat plate. We calculate the power spectra of fluctuations in thermal radiation recorded from a thin near-wall boundary layer for a wide range of initial flow parameters. The results show that changes in power spectra scaling can be directly related to the transitions in a flow pattern within the boundary layer.
\end{abstract}

\section{Introduction}

Liquid and gas jets impinging on a solid wall are ubiquitous both in nature and engineering. Jet systems provide effective way for momentum, heat and mass transfer and, thus, are widely used in industrial applications involving heating, cooling and drying processes. Moreover, jet and boundary layer flows are historically important for computational fluid dynamics (CFD), in particular as an excellent test case for validation of numerical codes and turbulence models. Impinging jets have been extensively studied, both numerically and experimentally, but most research has been focused on jet impingement heat transfer [1-3]. Particular attention has been given to jets impinging at short nozzle-to-plate distances when the highest heat/mass transfer rates are observed [4]. Impinging jets, however, are also of interest from a fundamental point of view as they provide a simple configuration to study shear and boundary layer flows.

Here, we use a configuration of a single round submerged water jet impinging on a flat plate to study the flow within a thin boundary layer using infrared (IR) thermography. The experimental technique is based on thermal imaging of fluctuations in infrared radiation emitted from turbulent water boundary layers $[5,6]$. Focusing an IR camera on the inner side of an IR-transparent window allows recording heat fluxes from a thin near-wall water layer since liquid water itself is highly absorptive in the mid-wave IR waveband.

\section{Experimental procedure and methodology}

In the present experiments, a hot jet submerged in a $25 \times 25 \times 30 \mathrm{~cm}^{3}$ cold-water tank impinges normally on a vertical flat tank wall. The jet is issued from a circular nozzle of diameter $D=1-4 \mathrm{~mm}$. The distance between the nozzle and the impingement plate is $\mathrm{H}=1-12 \mathrm{~mm}$. The impingement plate is a window of calcium fluoride $\left(\mathrm{CaF}_{2}\right), 2-\mathrm{mm}$ thick and $50-\mathrm{mm}$ in diameter. $\mathrm{CaF}_{2}$ is a widespread and inexpensive material providing, however, excellent transmittance (more than $90 \%$ ) of mid-wave IR radiation.

Fig. 1a shows a schematic representation of the flow. As the jet approaches the impingement plate, it decelerates until a stagnation point, where the streamwise mean velocity is zero. Then the flow accelerates radially forming a wall jet, which is composed of a boundary-like region surrounded by a free mixing layer [1-4]. In experiments, different flow parameters are tested - nozzle-to-plate spacings (H/D) and jet exit velocities $v_{\mathrm{j}}$ (jet Reynolds numbers $R e_{\mathrm{j}}$ ).

The thermal imaging of the impinging process is performed using a 3.7-4.8 $\mu$ m (mid-wave) IR camera FLIR SC7000 (as sketched in Fig 1b). The thermal videos are recorded at frame rates from $115 \mathrm{~Hz}$ (at full $640 \times 512$ pixel resolution) up to $400 \mathrm{~Hz}$ (with limited resolution). Most of the present thermal images are recorded at a rate of $300 \mathrm{~Hz}$, which, according to the Nyquist theory, enables observation of flow phenomena with frequencies up to $150 \mathrm{~Hz}$. Images captured using a 50-mm lens have a spatial resolution of $0.15 \mathrm{~mm} /$ pixel. The camera is focused on the inner surface of the $\mathrm{CaF}_{2}$ impingement plate.

Fig. 2 shows example instantaneous and time-averaged thermal images of the impinging jet. Substituting the relevant values of the absorption coefficient into the Bouguer law, we estimate that $99 \%$ of the infrared radiation within the operating spectral range of the camera is absorbed in a water layer as thin as $0.5 \mathrm{~mm}$. Thus, the thermal fluctuations captured by the IR camera are emitted from a sub-millimeter boundary layer.

The processing of the obtained thermal data includes the following stages:

1) points on each time-evolving temperature map at different locations from the stagnation point $(R)$ are selected;

2) the time dependences of measured thermal signals at each point are extracted using the FLIR software; algorithm;

3) the energy power spectra for each time-series are calculated using the Fast Fourier Transform (FFT) 
4) the characteristic frequencies of temperature pulsations and the power laws of the obtained power spectra are determined.

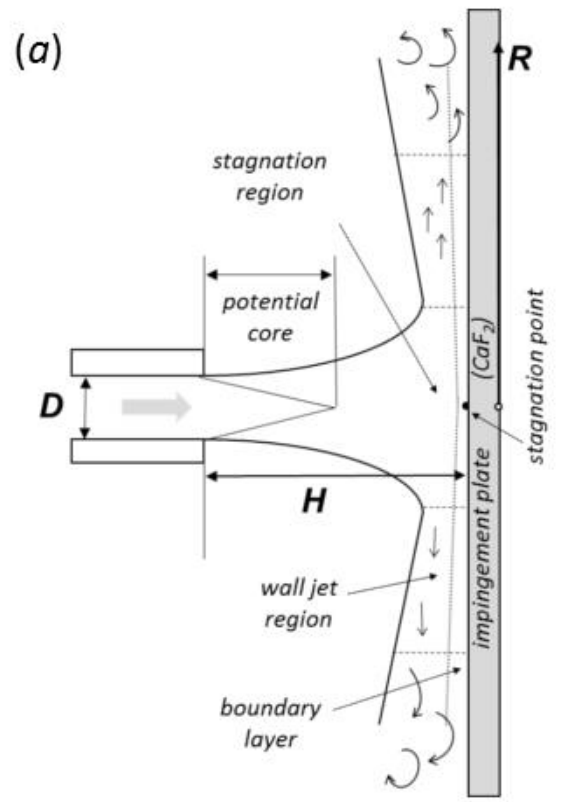

Fig. 1. A flow schematic (a) and a sketch of the experimental setup (b)

The main requirement for the thermal imaging to be applied for the spectral analysis is that the temperature can be treated as a passive tracer not affecting the fluid motion. In the experiments, we select an optimal recording duration of $1-2 \mathrm{~s}$ (corresponding to 256 or 512 thermal frames), which is less than typical thermal conduction time scales. Fig. $2 \mathrm{~b}$ shows example temperature time series at three different distances $(R)$ from the stagnation point (Fig. 2a). During the selected time interval, we can attribute the observed temperature fluctuations to the mixing process of fluid elements of constant temperatures ("thermal spots").
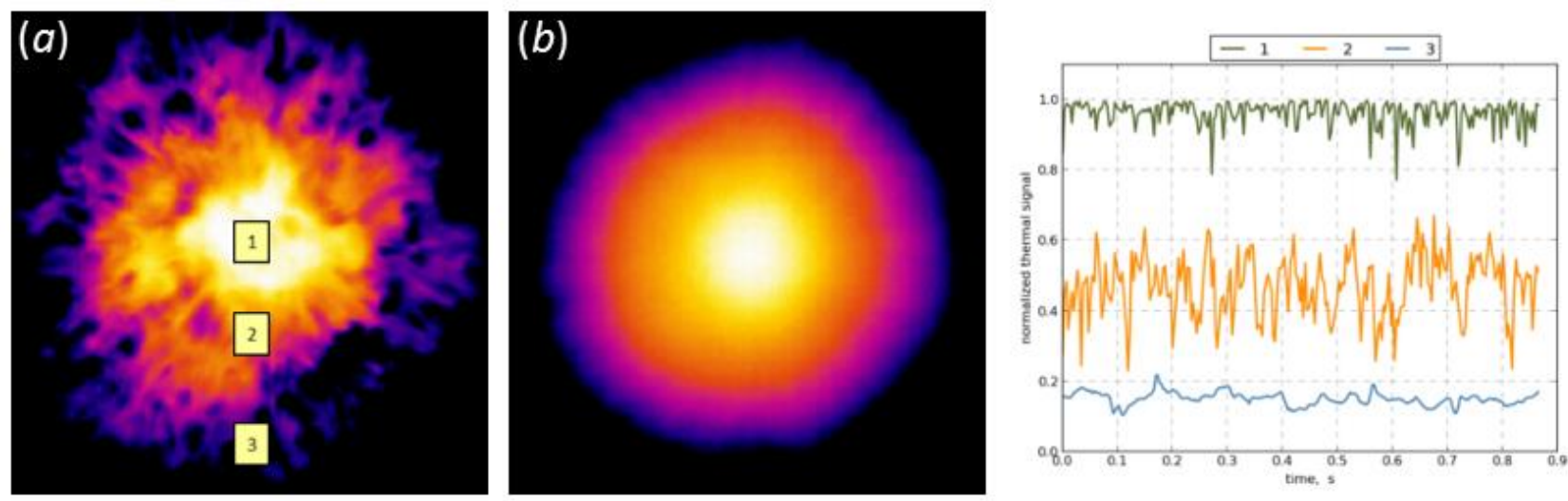

Fig. 2. Instantaneous (a) and time-averaged (b) thermal images of an impinging jet and time series of a normalized thermal signal at three selected points (c).

\section{Results and discussion}

First, we study the contribution of the thermal noise to the spectra of the obtained thermal signals. Fig. 3a shows the power spectra of two signals: one at $R \sim 5 \mathrm{~mm}$ from the stagnation point and one away from the impingement region where the fluctuations in thermal signal are mostly due to the camera noise. The results indicate that the measurement noise affects the signal spectra only for frequencies above $80-90 \mathrm{~Hz}$.

Another important aspect to consider is the effect of the temperature difference between the hot jet and cold ambient water, $\Delta T$. Varying the value of $\Delta T$ in the range of 5 to $25{ }^{\circ} \mathrm{C}$ with other jet parameters fixed, we find that the thermal pulsations at the same distances from the stagnation point exhibit similar spectral behavior. Moreover, spectra 
obtained for a cold jet submerged into a warm water tank are found to clearly follow the same scaling as for a hot jet submerged into a cold water tank (Fig. 3b). This observation supports the role of temperature as a passive tracer in the studied flow. The values of $\Delta \mathrm{T}$ at least $5-10{ }^{\circ} \mathrm{C}$ or higher, however, are preferable to achieve the desired contrast in thermal images.

While the spectral behavior of thermal fluctuations at specified points within the boundary layer is not affected by $\Delta \mathrm{T}$, we observe large changes in the power spectra depending on the distance from the stagnation point. Fig. 4 presents the example spectra of thermal fluctuations at three distances from the stagnation point $\left(D=3 \mathrm{~mm}, H / D=3, v_{j}\right.$ $\left.=2.4 \mathrm{~m} / \mathrm{s}, R e_{j}=7700\right)$. The power spectral density around the stagnation point $(r / D=0$, point 1 in Fig. $2 a)$ is typically almost constant (frequency-independent). At distances around R/D = 4 (point 2 in Fig. 2a) the power law starts following the classical Kolmogorov "-5/3" power law [7]. Further from the stagnation region ( $r / D \sim 8$, point 3 in Fig. 2a) the power spectrum becomes steeper and a slope of approximately -3 is observed. Thus, fluctuations in thermal radiation emitted from particular regions within the thin water boundary layer possess certain spectral properties.
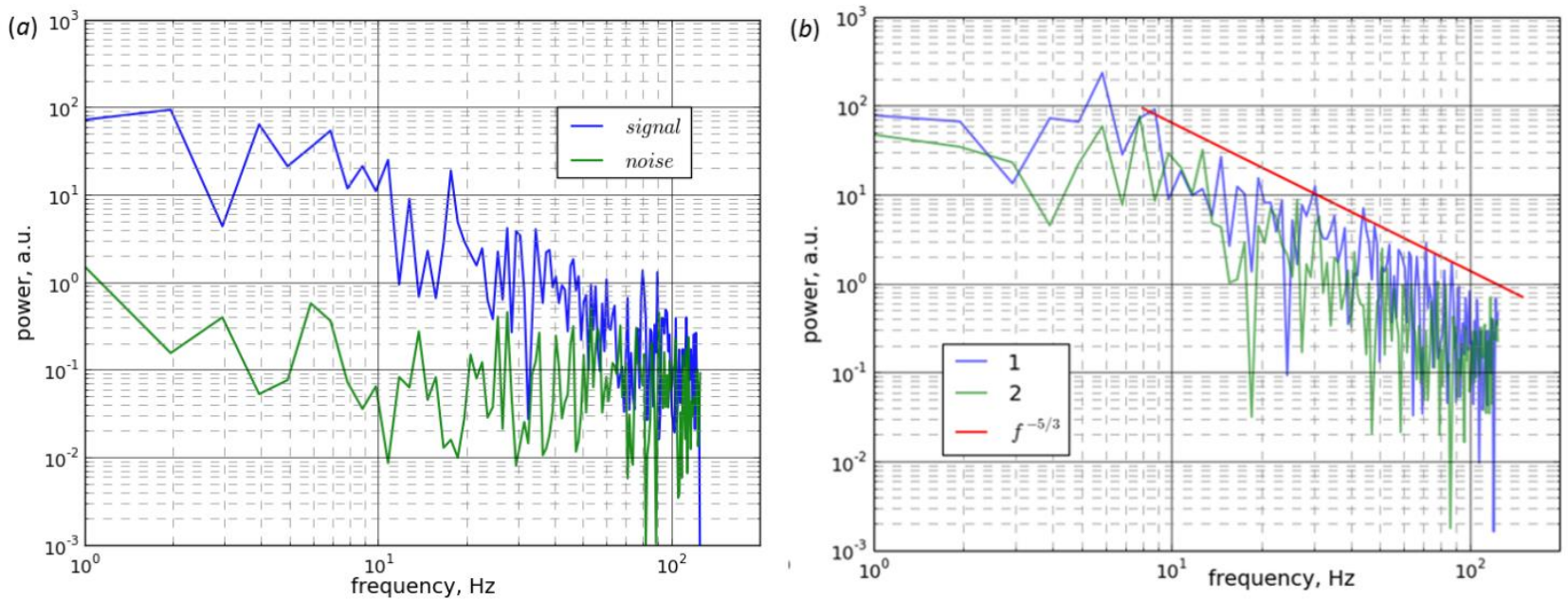

Fig. 3. Comparison of: (a) signal and noise spectra and (b) spectra obtained for fixed flow parameters at similar distances from the stagnation point $(R): 1$ - hot jet submerged in cold water $\left(\Delta T=25^{\circ} \mathrm{C}\right) ; 2$ - cold jet submerged in warm water $\left(\Delta T=15^{\circ} \mathrm{C}\right)$
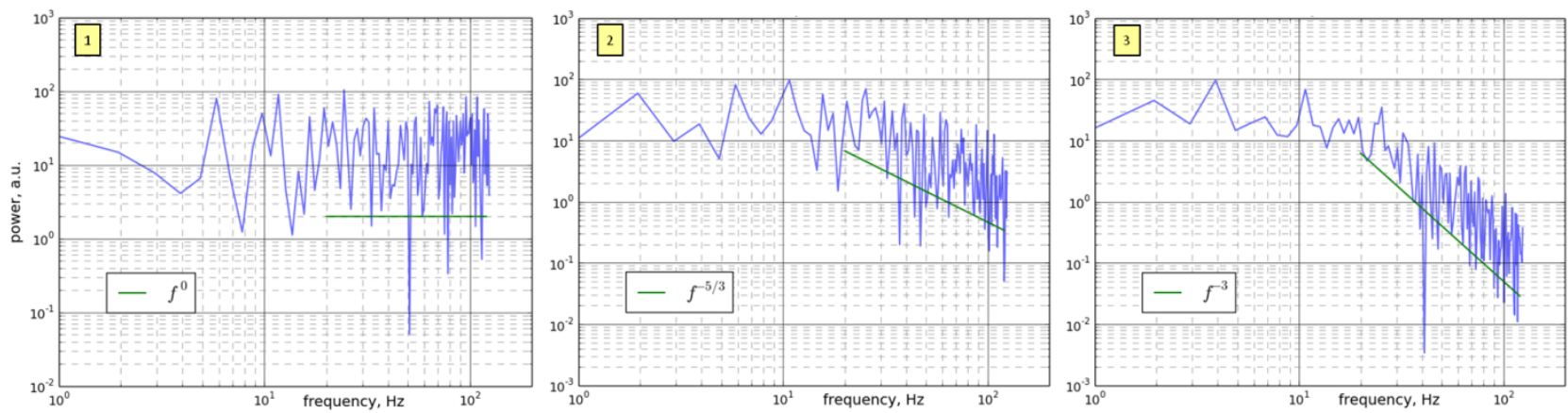

Fig. 4. Typical spectra of thermal pulsations at three selected points of the flow (Fig. 2$): 1-R / D=0 ; 2-R / D \sim$ $4 ; R / D \sim 8$

Generally, all the obtained spectra can be approximated with a power law in the form of $E \sim f^{a}$ within the frequency range of approx. 10-80 Hz. For each spectrum, we compute the value of a using the least mean square algorithm. Fig. 5 shows the dependence of the spectral scaling on the normalized distance from the stagnation point, $R / D$, for the jet nozzle diameter $D=3 \mathrm{~mm}$, nozzle-to-plate spacing $H / D=3$ and three values of the jet exit velocity. The vertical error is due to ambiguity in the estimation of the frequency range and averaging among several experimental data sets. The results indicate that the boundary layer flow appears to stay laminar within 3-4 diameters from the stagnation point, where there is no intensive mixing of "thermal spots" with ambient water. Further downstream, the power spectrum steepens and starts following the Kolmogorov $-5 / 3$ scaling. The higher the jet exit velocity (jet Reynolds number), the further from the stagnation point this transition takes place. After reaching the minimum value (as low as a $=-3$ for some flow configurations), the slope scaling tends to rise back reaching, again, near-zero values as in the stagnation area. Similar dependences are obtained for all jet geometries and exit velocities considered.

We analyze the transition to $f^{5 / 3}$ behaviour of the spectra, i.e. we calculate the positions at which the slope curves intersect the $\alpha=-5 / 3$ line (as in Fig. 5). Fig. 6 summarizes the obtained data for two fixed normalized nozzle-to- 
plate spacings, $H / D=2$ and $H / D=3$, showing the estimated position of the transition point (in normalized R/D values) versus the jet exit velocity. We find that the area of a laminar boundary layer flow around the stagnation point increases with increasing the jet velocity (the flow Reynolds number); however, this relationship is not linear. In general, the changes in the power spectrum scaling of captured thermal fluctuations appear to be directly related to the changes in the flow regime within a thin boundary layer. At locations where the thermal fluctuations penetrate the boundary layer, the power spectra follow a close to Kolmogorov scaling value for developed turbulence.

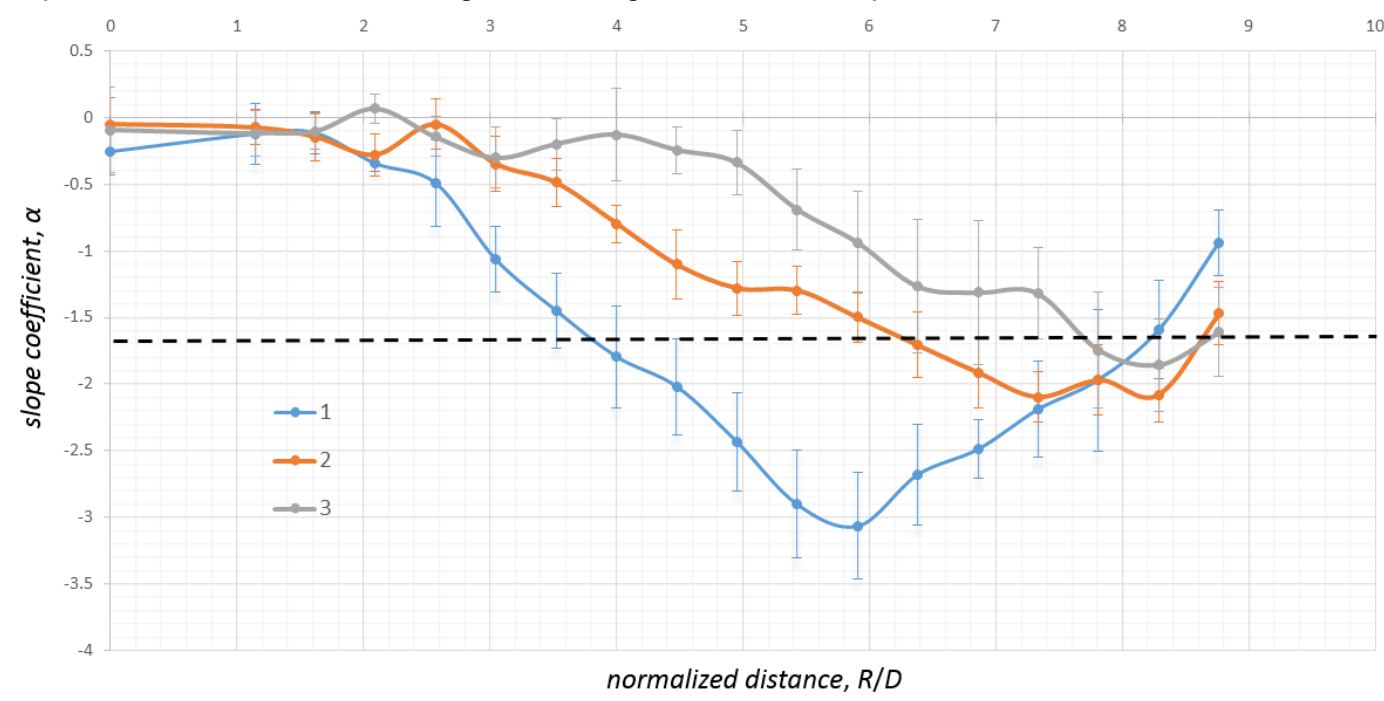

Fig. 5. The computed slope coefficient of the power spectrum depending on the normalized distance from the stagnation point $\left(D=2 \mathrm{~mm}, H / D=3\right.$ ) for three jet exit velocities: $1-v_{j}=2.9 \mathrm{~m} / \mathrm{s}, R e_{j} \sim 6000 ; 2-v_{j}=5.4 \mathrm{~m} / \mathrm{s}, R e_{j} \sim$ $11500 ; 3-v_{j}=7.6 \mathrm{~m} / \mathrm{s}, R_{j} \sim 16000$. Dashed line indicates the $\alpha=-5 / 3$ value.

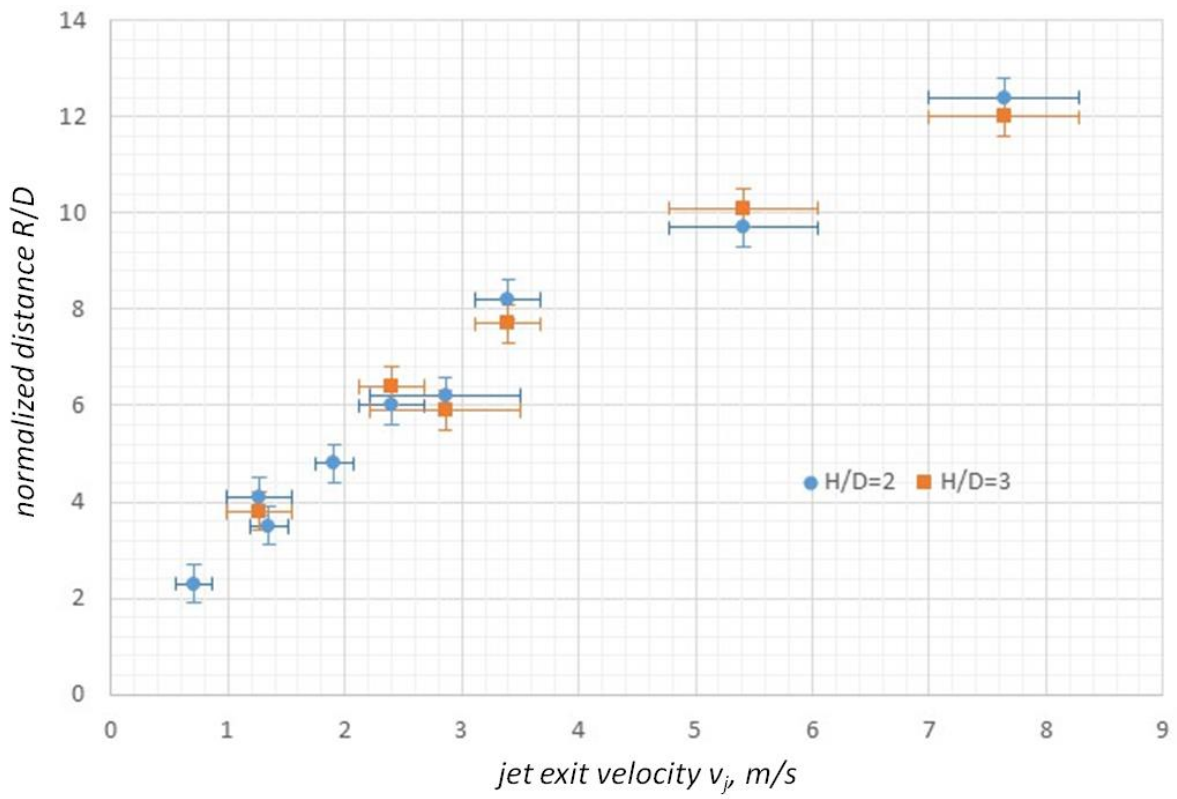

Fig. 6. Transition point of versus jet exit velocity for two values of nozzle-to-plate spacing: $H / D=2$ and $H / D=3$.

\section{Conclusions}

We have proposed and tested a new methodology for the quantitative analysis of fluid boundary layers based on high-speed thermographic measurements. The methodology has been applied to study a boundary layer flow induced by a submerged impinging water jet. The infrared data has been collected for a wide range of flow parameters: jet nozzle diameters $D=1-4$, nozzle-to-plate distances $H=1-6$, jet exit velocities $v=0.5-20 \mathrm{~m} / \mathrm{s}$ (corresponding to flow Reynolds numbers $\left.\sim 10^{3}-10^{5}\right)$. The analysis of the power spectra of the recorded thermal fluctuations has shown that the slope scaling changes are a function of the distance from the impingement point (i.e., the R/D values), and this function varies 
depending on initial flow parameters (such as the flow rate and the relative nozzle-to-plate distance, H/D). The changes in the spectrum scaling can be related to the flow pattern transitions within the boundary layer. Tracking these changes allows capturing different flow regimes of the wall jet. Thus, the proposed methodology based on high-speed IR thermography opens new possibilities for analysis and characterization of boundary layers of IR-absorptive fluids.

This work has been supported by Russian Foundation for Basic Research (Grant number 16-38-60186).

\section{REFERENCES}

[1] Grenson $\mathrm{P}$, Léon $\mathrm{O}$, Reulet $\mathrm{P}$, Aupoix $\mathrm{B}$. Investigation of an impinging heated jet for a small nozzle-to-plate distance and high Reynolds number: An extensive experimental approach. International Journal of Heat and Mass Transfer. 2016:102:801-815.

[2] Zuckerman N, Lior N. Jet impingement heat transfer: physics, correlations, and numerical modeling, Adv. Heat Transfer. 2006:39:565-631.

[3] Loureiro JBR, Silva Freire APS. Velocity and temperature profiles, wall shear stress and heat transfer coefficient of turbulent impinging jets. International Journal of Heat and Mass Transfer 2017:107:846-861.

[4] Carlomagno GM, laniro A, Thermo-fluid-dynamics of submerged jets impinging at short nozzle-to-plate distance: a review, Exp. Thermal Fluid Sci. 2014:58:15-35.

[5] Znamenskaya IA, Koroteeva EY. Time-resolved thermography of impinging water jet. Journal of Flow Visualization and Image Processing. 2013:20(1-2);25-33.

[6] Bolshukhin MA., Znamenskaya IA, Fomichev VI. A method of quantitative analysis of rapid thermal processes through vessel walls under nonisothermal liquid flow. Dokl. Phys. 2015:60:524-527.

[7] Kolmogorov AN. The local structure of turbulence in incompressible viscous fluid for very large Reynolds numbers. Proceedings of the Royal Society A.1991:434:9-13. 